\title{
A GENERALIZED CLAIRAUT'S THEOREM IN MINKOWSKI SPACE
}

\author{
ANSI SAAD AND ROBERT J. LOW
}

Communicated by Gregory Naber

\begin{abstract}
In Euclidean space, the geodesics on a surface of revolution can be characterized by means of Clairaut's theorem, which essentially says that the geodesics are curves of fixed angular momentum. A similar result is known for three dimensional Minkowski space for timelike geodesics on surfaces of revolution about the time axis. Here, we extend this result to consider generalizations of surfaces of revolution to those surfaces generated by any one-parameter subgroup of the Lorentz group. We also observe that the geodesic flow in this case is easily seen to be a completely integrable system, and give the explicit formulae for the timelike geodesics.
\end{abstract}

\section{Introduction}

The change of signature from Euclidean to Minkowskian geometry results in a fascinating interplay between the two forms of geometry: there exists a formal algebraic similarity in many aspects of the geometry, coupled to important differences between the two, especially in global situations. The lecture notes of López [4], for example, provide a detailed consideration of many of the aspects of three dimensional Minkowski space. The differences arise in various way, and we will here be concerned with some of the consequences of the fact that vectors in Minkowski space can be classified as timelike, null, or spacelike by means of the inner product. In a previous work [8] we considered surfaces of revolution in the situation with the closest analogy to the Euclidean situation, namely that of the timelike geodesics on surfaces obtained by rotating a timelike curve about the $t$-axis in Minkowski space. There are, of course, other types of surface of revolution in Minkowski space [3], and in this work we will extend our consideration to these other classes of surface. We will begin in Section 2 by briefly reviewing Clairaut's theorem in the Euclidean case (for a more detailed exposition, see, for example, Pressley's text [7], and for applications see $[1,5,6])$, and the situation for rotations about the $t$-axis in Minkowski space. In Section 3 we see how there are three different types of oneparameter subgroup of the three dimensional Lorentz group, giving rise to three different class of surface of revolution. In Section 4 we establish an analogue of 
Clairaut's theorem in each case. Finally, in Section 5, we note that the geodesics are, in each case, determined by a completely integrable system of equations, and obtain the explicit formulae for the geodesics in terms of quadratures.

\section{Clairaut's Theorem}

We begin by setting up some notation, which will be useful in both the Euclidean and the Minkowskian cases. For reference, we will equip three dimensional Euclidean space $\mathbb{E}^{3}$ with coordinates $(x, y, z)$, the standard basis $\left\{\mathbf{e}_{x}, \mathbf{e}_{y}, \mathbf{e}_{z}\right\}$ and the metric $\mathrm{d} x^{2}+\mathrm{d} y^{2}+\mathrm{d} z^{2}$, and three dimensional Minkowski space $\mathbb{M}^{3}$ with coordinates $(x, y, t)$, the standard basis $\left\{\mathbf{e}_{x}, \mathbf{e}_{y}, \mathbf{e}_{t}\right\}$ and metric $\mathrm{d} x^{2}+\mathrm{d} y^{2}-\mathrm{d} t^{2}$.

Let $\Sigma$ be a surface of revolution in $\mathbb{E}^{3}$, obtained by rotating the curve $x=\rho(u), y=$ $0, z=h(u)$ about the $z$-axis, where we assume that $\rho>0$ and that $\rho^{\prime}(u)^{2}+$ $h^{\prime}(u)^{2}=1$. Then $\Sigma$ is parameterized by

$$
\mathbf{x}(u, v)=\left[\begin{array}{c}
\rho(u) \cos (v) \\
\rho(u) \sin (v) \\
h(u)
\end{array}\right]
$$

and has first fundamental form

$$
I=\left[\begin{array}{cc}
\mathbf{x}_{u} \cdot \mathbf{x}_{u} & \mathbf{x}_{u} \cdot \mathbf{x}_{v} \\
\mathbf{x}_{v} \cdot \mathbf{x}_{u} & \mathbf{x}_{v} \cdot \mathbf{x}_{v}
\end{array}\right]=\left[\begin{array}{cc}
1 & 0 \\
0 & \rho(u)^{2}
\end{array}\right] .
$$

We also have

$$
\mathbf{x}_{u}=\mathbf{n}_{u} \quad \text { and } \quad \mathbf{x}_{v}=\rho \mathbf{n}_{v}
$$

where $\mathbf{n}_{u}$ is the unit vector pointing along meridians of $\Sigma$ and $\mathbf{n}_{v}$ is the unit vector pointing along parallels of $\Sigma$. Since $\mathbf{n}_{u} \cdot \mathbf{n}_{v}=0$, the two form an orthonormal basis for the tangent plane at each point, and an arbitrary unit tangent vector vector $\mathbf{t}$ is therefore of the form $\mathbf{n}_{u} \cos \theta+\mathbf{n}_{v} \sin \theta$ where $\theta$ is the angle between $\mathbf{t}$ and $\mathbf{n}_{u}$.

From the first fundamental form, we have the Lagrangian

$$
\frac{1}{2}\left(\dot{u}^{2}+\rho^{2} \dot{v}^{2}\right)
$$

and so the Euler-Lagrange equations, whose solutions are arc-length parameterised geodesics, are

$$
\ddot{u}=\rho \rho^{\prime} \dot{v}^{2}, \quad \frac{\mathrm{d}}{\mathrm{d} s}\left(\rho^{2} \dot{v}\right)=0 .
$$

But now, if $\gamma(\mathbf{s})$ is an arc-length paramaterised geodesic given by $(u(s), v(s))$ we also have

$$
\dot{\gamma}=\dot{u} \mathbf{n}_{u}+\rho \dot{v} \mathbf{n}_{v}=\mathbf{n}_{u} \cos \theta+\mathbf{n}_{v} \sin \theta
$$


where $\theta$ is the angle between $\dot{\gamma}$ and a meridian.

Equating the components of $\mathbf{n}_{v}$ in the latter two expressions, we see that $\rho \dot{v}=$ $\sin \theta$, so that $\rho^{2} \dot{v}=\rho \sin \theta$, and hence the second Euler-Lagrange equation is equivalent to $\rho \sin \theta$ being a constant along $\gamma$.

Conversely, if $\gamma$ is a unit speed curve with $\rho \sin \theta$ constant, the second EulerLagrange equation is immediately satisfied: next, differentiating $\dot{u}^{2}+\rho^{2} \dot{v}^{2}=1$ and substituting this into the second Euler-Lagrange equation yields the first EulerLagrange equation.

This establishes Clairaut's theorem, and we observe in passing that all meridians are geodesics.

If we now replace the $z$-axis of $\mathbb{E}^{3}$ by the $t$-axis of $\mathbb{M}^{3}$, the parameterisation of the surface and the corresponding analysis are formally almost identical, where we take $\rho^{\prime}(u)^{2}-h^{\prime}(u)^{2}=-1$ so that the generating curve is timelike, resulting in a surface of revolution whose induced metric is Lorentzian.

We now have orthogonal unit timelike and spacelike vectors $\mathbf{n}_{u}$ and $\mathbf{n}_{v}$ given by $\mathbf{x}_{u}=\mathbf{n}_{u}$ and $\mathbf{x}_{v}=\rho \mathbf{n}_{v}$ respectively, so that a unit timelike tangent vector is given by $\mathbf{t}=\mathbf{n}_{u} \cosh (\theta)+\mathbf{n}_{v} \sinh (\theta)$, where $\theta$ is the hyperbolic angle between $\mathbf{t}$ and $\mathbf{n}_{u}$. Repeating the previous argument then tells us that timelike geodesics in the surface of revolution are characterised by the constant $\rho \sinh (\theta)=\rho^{2} \dot{v}$.

Note in passing that if we were to choose a spacelike generating curve with $\rho^{\prime}(u)^{2}+$ $h^{\prime}(u)^{2}=1$, the metric induced on the tangent plane is Riemannian, and the situation for spacelike geodesics then becomes identical to that the Euclidean case.

\section{Surfaces of Revolution in Minkowski Space}

In the previous section we considered surfaces invariant under the action of a oneparameter subgroup of the symmetry group of space, which in each case was isomorphic to $\mathrm{SO}(2)$, and could be regarded as the usual rotations about the origin in surfaces of constant $z$, or $t$, according as we consider the Euclidean or Minkowskian case. In the Euclidean case, there is no other possibility: all oneparameter subgroups of $\mathrm{SO}(3)$ are of this form, and by an appropriate choice of coordinates consist of rotations fixing the $z$-axis. The situation is, however, rather different in the Minkowskian case.

Let us denote by $\Lambda$ an arbitrary member of the Lorentz group $\mathrm{SO}(2,1)$, and by $\Lambda(v)$ a curve in $\mathrm{SO}(2,1)$ such that $\Lambda(0)$ is the identity. Differentiating the relationship $\Lambda(v)^{T} \eta \Lambda(v)=\eta$, where $\eta=\operatorname{diag}(1,1,-1)$ and setting $v=0$ we find that $\lambda=$ 
$\dot{\Lambda}(0)$ must be of the form

$$
\lambda=\left[\begin{array}{rrr}
0 & \alpha & \beta \\
-\alpha & 0 & \gamma \\
\beta & \gamma & 0
\end{array}\right]
$$

For any choice of $\alpha, \beta, \gamma$ this matrix has zero determinant, and so an eigenvector of eigenvalue 0 . It follows then that the corresponding one-parameter subgroup of $\mathrm{SO}(2,1)$ given by $\exp (v \lambda)$ fixes pointwise the line through the origin determined by this eigenvector, and so can be regarded as a one-parameter group of rotations with this line as axis of rotation.

We observe that the vector $\mathbf{e}_{t}$ is an eigenvector with eigenvalue 0 if $\beta=\gamma=0$, and so we can take

$$
\lambda=\left[\begin{array}{rrr}
0 & 1 & 0 \\
-1 & 0 & 0 \\
0 & 0 & 0
\end{array}\right]
$$

giving

$$
\exp (v \lambda)=\left[\begin{array}{ccc}
\cos (v) & \sin (v) & 0 \\
-\sin (v) & \cos (v) & 0 \\
0 & 0 & 1
\end{array}\right]
$$

This case is therefore the previously considered one of rotations about the $t$-axis. Similarly, $\mathbf{e}_{y}$ is an eigenvector with eigenvalue 0 if $\alpha=\gamma=0$ so that we can take

$$
\lambda=\left[\begin{array}{lll}
0 & 0 & 1 \\
0 & 0 & 0 \\
1 & 0 & 0
\end{array}\right] \text { and } \operatorname{so} \exp (v \lambda)=\left[\begin{array}{ccc}
\cosh (v) & 0 & \sinh (v) \\
0 & 1 & 0 \\
\sinh (v) & 0 & \cosh (v)
\end{array}\right] .
$$

For convenience we denote by $B_{y}$ the group of boosts fixing the $y$-axis, and let $B_{y}(v)=\exp (v \lambda)$.

Finally, $\mathbf{e}_{y}+\mathbf{e}_{t}$ is an eigenvector with eigenvalue 0 if $\alpha=-\beta, \gamma=0$, giving

$$
\lambda=\left[\begin{array}{rrr}
0 & 1 & -1 \\
-1 & 0 & 0 \\
-1 & 0 & 0
\end{array}\right] \text { and so } \exp (v \lambda)=\left[\begin{array}{ccc}
1 & v & -v \\
-v & 1-v^{2} / 2 & v^{2} / 2 \\
-v & -v^{2} / 2 & 1+v^{2} / 2
\end{array}\right] .
$$

Similarly to the previous case, denote by $B_{n}$ the group of null rotations fixing the axis given by $\mathbf{e}_{y}+\mathbf{e}_{\mathbf{t}}$, and let $B_{n}(v)=\exp (v \lambda)$.

Since every vector is spacelike, null, or timelike, any other one-parameter subgroup of $\mathrm{SO}(2,1)$ can be put into this form by an appropriate choice of axes. We therefore consider those surfaces which are invariant under the action of each of these groups and have a Lorentz metric induced on them, and will investigate the timelike geodesics on each surface. 
We omit the case of the timelike axis of rotation, as this has been considered already. This leaves the two cases of a spacelike axis of rotation (corresponding to the one-parameter group of boosts $B_{y}$ ) and a null axis of rotation (corresponding to the one-parameter group of null rotations $B_{n}$ ).

\subsection{Surfaces of Revolution About a Spacelike Axis of Rotation}

Now, let $(x, y, t)$ be a point on a surface, $\Sigma$, invariant under the action of $B_{y}$. If $|x|<|t|$ then the orbit of $B_{y}$ on which $(x, y, t)$ lies is spacelike, and intersects the $y t$ plane at some unique value of $v$. Assume for the moment that $\Sigma$ lies entirely in the region $|x|<|t|$. Then the intersection of $\Sigma$ with the $y t$ plane is a generating curve for $\Sigma$. The tangent to any orbit is orthogonal to the $y t$ plane at the point of intersection; the tangent plane to $\Sigma$ at the point of intersection cannot be spacelike, and so neither can the tangent to the generating curve (since any vector space spanned by orthogonal spacelike vectors is itself spacelike). Thus $\Sigma$ is generated by a timelike curve in the $y t$ plane, given by $(0, \rho(u), h(u))$ with $u$ chosen so that $h^{\prime}(u)^{2}-\rho^{\prime}(u)^{2}=1$.

By the same type of argument, if $|x|>|t|$ the orbits are timelike, and assuming that $\Sigma$ lies in the region $|x|>|t|, \Sigma$ is generated by a spacelike curve lying in the $x y$ plane, which we take to be given by $(\rho(u), h(u), 0)$ where now $\rho^{\prime}(u)^{2}+h^{\prime}(u)^{2}=1$. There are therefore two simple classes of surface of revolution generated by $B_{y}$, one confined to the region $|x|<|t|$, and the other to the region $|x|>|t|$. The first has a timelike generating curve in the $y t$ plane, and the second has a spacelike generating curve in the $x y$ plane. The first fundamental forms of these two (in terms of the coordinates $(u, v))$ are, respectively

$$
\left[\begin{array}{cc}
-1 & 0 \\
0 & \rho^{2}(u)
\end{array}\right] \quad \text { and } \quad\left[\begin{array}{cc}
1 & 0 \\
0 & -\rho^{2}(u)
\end{array}\right] \text {. }
$$

\subsection{Surfaces of Revolution About a Null Axis of Rotation}

Again, let $(x, y, t)$ be a point on a surface, $\Sigma$, which is now invariant under the action of $B_{n}$. This time we assume that the surface does not intersect the plane $y=t$. Each point on $\Sigma$ lies on a curve passing through the $y t$ plane, and so $\Sigma$ is generated by a curve in the $y t$ plane. In this case the orbits are spacelike, and so the generating curve must be timelike. We take it to be of the form $(0, q(u), h(u))$ where $h^{\prime}(u)^{2}-q^{\prime}(u)^{2}=1$. The fundamental form is now

$$
\left[\begin{array}{cc}
-1 & 0 \\
0 & \rho^{2}(u)
\end{array}\right]
$$


where $\rho(u)=q(u)-h(u)$.

\subsection{Parallels and Meridians}

We generalize from the Euclidean situation the terminology of parallels and meridians. Here, a meridian is the image of the generating curve under the action of an element of $B_{y}$ or $B_{n}$, and a parallel is an orbit of the group action. Note that in the two cases where the generating curve is timelike, we have timelike meridians and spacelike parallels, but in the case where the generating curve is spacelike, it is the parallels which are timelike and the meridians which are spacelike.

\section{Generalizations of Clairaut's Theorem}

Now, the general point on any of our surfaces is given by $\mathbf{x}(u, v)$, which is the image under the appropriate group element with parameter $v$ of the generating curve element with parameter $u$. A curve $\gamma(s)$ on the surface is given by a curve $(u(s), v(s))$, and we assume that the parameter is chosen so that $\gamma$ is parameterised by proper time.

In each case of timelike meridians, the Lagrangian is given by

$$
\frac{1}{2}\left(-\dot{u}^{2}+\rho(u)^{2} \dot{v}^{2}\right)
$$

which is independent of $v$, so that $\rho^{2}(u) \dot{v}$ is a conserved quantity along the geodesic. We also have in each case

$$
\mathbf{x}_{u}=\mathbf{n}_{u}, \quad \mathbf{x}_{v}=\rho \mathbf{n}_{v}
$$

where $\mathbf{n}_{u}$ and $\mathbf{n}_{v}$ are orthogonal unit vectors pointing along meridians and parallels respectively, so that $\mathbf{n}_{u}$ is timelike and $\mathbf{n}_{v}$ is spacelike.

Furthermore, differentiating $\gamma$ gives

$$
\dot{\gamma}(s)=\mathbf{n}_{u} \dot{u}+\mathbf{n}_{v} \rho \dot{v}=\mathbf{n}_{u} \cosh (\theta)+\mathbf{n}_{v} \sinh (\theta)
$$

where $\theta$ is the hyperbolic angle between the tangent to $\gamma$ and the meridian.

We therefore see that in these cases the quantity $\rho \dot{v}^{2}=\rho \sinh (\theta)$ is conserved along every timelike geodesic, where $\theta$ is the hyperbolic angle between the geodesic and a meridian.

In the case of spacelike meridians, the Lagrangian becomes

$$
\frac{1}{2}\left(-\dot{u}^{2}+\rho(u)^{2} \dot{v}^{2}\right)
$$


which again gives the conserved quantity $\rho^{2} \dot{v}$.

This time, however, it is $\mathbf{n}_{u}$ that is spacelike and $\mathbf{n}_{v}$ that is timelike, and so we have the timelike vector $\dot{\gamma}$ given by

$$
\dot{\gamma}(s)=\mathbf{n}_{u} \dot{u}+\mathbf{n}_{v} \rho \dot{v}=\mathbf{n}_{u} \sinh (\phi)+\mathbf{n}_{v} \cosh (\phi)
$$

where $\phi$ is now the hyperbolic angle between the geodesic and a parallel.

In this case, the conserved quantity is therefore $\rho \cosh (\phi)$. As there is no analogue to the trigonometric notion of complementary angle, we cannot express this as $\rho \sinh (\theta)$ for some angle: however, we note that in the Euclidean case the quanties $\rho \sin (\theta)$, where $\theta$ is the angle between the geodesic and a meridian, and $\rho \cos (\phi)$, where $\phi$ is the angle between the geodesic and a parallel, are equal. Thus in this case also, the conserved quantity is the hyperbolic equivalent of the Euclidean conserved quantity.

It is then a simple algebraic exercise to check that in each of the three cases, conservation of the conserved quantity along a curve parameterised by proper time implies that the curve is a timelike geodesic, completing the analogue of Clairaut's theorem in each case.

It thus follows that for all three classes of surface of revolution, whether generated by rotations, boosts, or null rotations, Clairaut's theorem holds in the sense that the natural quantity corresponding to $\rho \sin (\theta)$ is constant along geodesics, and the value of this constant determines the timelike geodesics on the surface.

\section{Integrability}

As a final comment, we note that in each case we have two conserved quantities, namely the Lagrangian and $\rho \dot{v}^{2}$. Re-expressing this in terms of the Hamiltonian formalism, the conserved quantities are $p_{v}$, the momentum conjugate to $v$, and $H$, the Hamiltonian; since all conserved quantities Poisson commute with the Hamiltonian, and the two are functionally independent, we know from the Arnol'dLiouville theorem [2] that the geodesics can in principle be expressed in terms of quadratures.

In this case, we have

$$
-\dot{u}^{2}+\rho(u)^{2} \dot{v}^{2}= \pm 1, \quad \rho(u)^{2} \dot{v}=L
$$

where the sign on the right hand side of the first equation is positive in the case of the $B_{y}$-invariant surface generated by a spacelike curve, and negative otherwise, and $L$ is the value of the conserved quantity specifying a particular geodesic. 
From the conserved quantity, we obtain

$$
\dot{v}=L / \rho(u)^{2}
$$

so that

$$
\dot{u}^{2}=L^{2} / \rho(u)^{2} \mp 1
$$

giving

$$
\int \frac{\mathrm{d} u}{\sqrt{L^{2} / \rho(u)^{2} \mp 1}}=\int \mathrm{d} s .
$$

This gives (in principle) $u(s)$, so that we can finally obtain $v(s)$ from

$$
v=\int \frac{L}{\rho(u(s))^{2}} \mathrm{~d} s .
$$

\section{Conclusion}

We have shown that the geometrical analogy between Euclidean and Minkowski space obtained by replacing the trigonometric functions by hyperbolic ones also extends to Clairaut's theorem, not only in the obvious case of surfaces generated by rotations about the time axis, but also in the cases of surfaces generated by boosts and null rotations. We also find the geodesics on such a surface of rotation in terms of quadratures.

\section{Acknowledgements}

Ansi Saad thanks Omar Al-mukhtar University, in Albaida - Libya for financial support. Both authors thank Professor Lionel Mason for his suggestions for improvement to an earlier version of this work.

\section{References}

[1] Alexander J., Closed Geodesics on Certain Surfaces of Revolution, J. Geom. Symmetry Phys. 8 (2006) 1-16.

[2] Arnol'd V., Mathematical Methods of Classical Mechanics, $2^{\text {nd }}$ Edn, Springer, New York 1989.

[3] Kazan A. and Karadag H., A Classification of Surfaces of Revolution in Lorentz-Minkowski Space, International Journal of Contemporary Mathematical Sciences 6 (2011) 1915-1928. 
[4] López R., Differential Geometry of Curves and Surfaces in LorentzMinkowski Space, Int. Electron. J. Geom. 7 (2014) 44-107.

[5] Mladenov I. and Oprea J., The Mylar Ballon Revisited, Am. Math. Monthly 110 (2003) 761-784.

[6] Mladenov I. and Oprea J., Unduloids and Their Closed Geodesics, Geometry, Integrability \& Quantization 4 (2003) 206-234.

[7] Pressley A., Elementary Differential Geometry, Springer, Great Britain 2010.

[8] Saad A. and Low R., Clairaut's Theorem in Minkowski Space, J. Geom. Symmetry Phys. 28 (2012) 105-112.

Ansi Saad

Department of Mathematics

Omar Al-Mukhtar University

B.O. Box: 919 Albaida

Albaida, Libya

Email address: anissaadeout look.com

Robert J. Low

Department of Mathematics and Physics

Coventry University

Coventry CV1 5FB, UK

Email address: mtx014@coventry.ac.uk 